\title{
Sonographic appearance of Krukenberg tumors: a rare case report
}

\author{
Iuliia Naumova ${ }^{1}$, Camil Castelo-Branco² \\ ${ }^{1}$ Department of Obstetrics and Gynecology, Faculty of General Medicine Saratov State Medical University named after V.I. Razumovsky, Saratov, Russia \\ ${ }^{2}$ Clinic Institute of Gynecology, Obstetrics and Neonatology, Hospital Clinic-Institut d'Investigacions Biome`diques August Pi i Sunyer (IDIBAPS), University \\ of Barcelona, Barcelona, Spain
}

Received: 02 January 2020 / Accepted: 10 March 2020

\begin{abstract}
Kruckenberg tumor is a metastatic lesion of the ovary, the source of which is the primary cancerous focus in other organs (generally in the digestive system); it often causes difficulties in differential diagnosis with primary ovarian cancer. We report a case of a 67-yearold woman who presented bilateral, $3 \mathrm{~cm}$ in diameter, pure solid, encapsulated and hyperechoic smooth masses, surrounded by a hypoechoic ring with clear tumor margins. Doppler ultrasonography revealed abundant vascularization, with a characteristic treeshaped structure penetrating from the periphery into the central part of the lesion with low impedance and diminished vascular resistance. Approximately $5 \%$ to $10 \%$ of all ovarian malignancies are metastatic carcinomas. Asymmetrically enlarged bilateral encapsulated masses, with variable intratumoral echogenic density, and ascites are the main characteristics of sonography of Krukenberg tumors. The structure of these tumors is usually hyperechoic with abundant vascularization. Several sonographic markers have been used to show the low impedance of intratumoral circulation in ovarian malignancy, attributing this to the active angiogenesis of these tumors. Krukenberg tumors have a poor prognosis, with an overall 5 -year survival rate of $12 \%$. Hence prophylactic bilateral oophorectomy is recommended for women with stomach cancer.
\end{abstract}

Keywords: gastric carcinoma, Krukenberg tumors, ultrasound

\section{Introduction}

It has been more than 100 years since Friedrich E. Krukenberg, a German pathologist, originally recognized six patients with unusual ovarian fibrosarcomas with elements resembling carcinoma [1]. Later on, Schlaggenhoffer reinterpreted this entity as a metastatic sarcomatous-like, signet ring cell with intracellular mucin tumor of the ovary [2].

Krukenberg tumors are defined as the presence of any metastasis to the ovaries. Among the primary lesions in the gastrointestinal tract, the colon is the most common site in western countries, and the stomach is the most common site in Asian countries [3]. Gastric cancer has been reported as the most frequent primary source responsible for approximately 50-70\% of these tumors [4]. The estimated incidence of these tumors is approximately $0.6 \times 100000$ per year [5]. The mean age of the patients who present this type of tumor is $45 \pm 10$ years 
due to the rich vascular flow in the ovaries of young women. It is believed that Krukenberg tumor metastases occur via blood flow, although it has been stated that when this type of tumor is detected, more than half of the patients have ascites; suggesting that peritoneal spread may be another important route of metastasis.

More than $\mathbf{8 0} \%$ of cases of Krukenberg tumors are bilateral and are typically solid with various shapes. Size is variable and sometimes exceeds $10 \mathrm{~cm}$, although stomach metastasis is usually smaller than bowel metastasis. Clinical symptoms of these tumors are exclusively related to the ovary. Around $90 \%$ present abdominal pain and swelling whereas genital bleeding and virilization are uncommon complaints.

\section{Case}

A 67-year-old patient diagnosed with gastric carcinoma in January 2011 underwent subtotal gastrectomy and lymphadenectomy. Histopathology confirmed gastric adenocarcinoma stage IIA; therefore, combined radiation and chemotherapy were offered to the patient, but she refused any additional treatments. Since then, follow-up with computed tomography (CT) plus endoscopy was performed every six months. In July 2015, a control CT scan showed a $6 \mathrm{~cm}$ tumor on the anterior abdominal wall at the epigastrium, which was surgically removed. Pathology confirmed a locoregional recurrence of gastric adenocarcinoma. Four months later, bilateral, $3 \mathrm{~cm}$ in diameter, kidneyshaped, pure solid, smooth, encapsulated ovaries were observed; with a hyperechogenic center and a hypoechogenic ring [Figure 1]. Clear tumor margins were identified. Color Doppler examination showed abundant vascularization with a characteristic tree-shaped structure penetrating into the central part of the lesion [Figure 2].

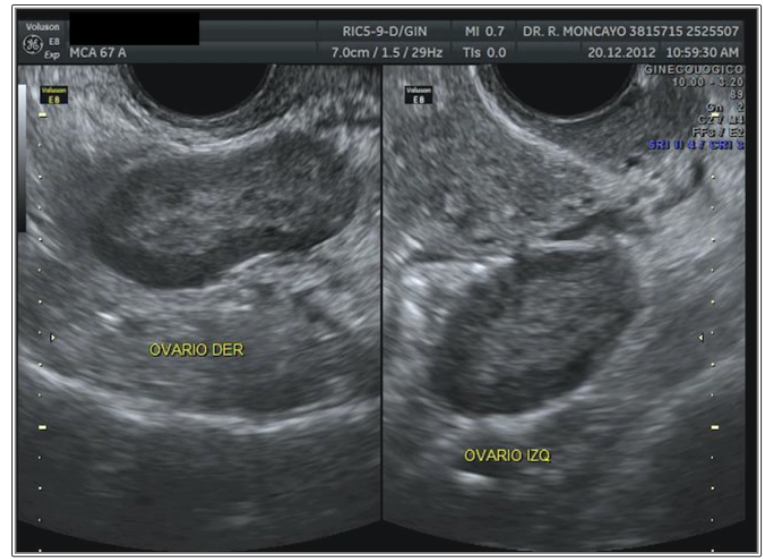

Figure 1. Ultrasound appearance of Krukenberg tumors: kidney-shaped, pure solid, smooth, encapsulated with a hyperechogenic center and a hypoechogenic ring.

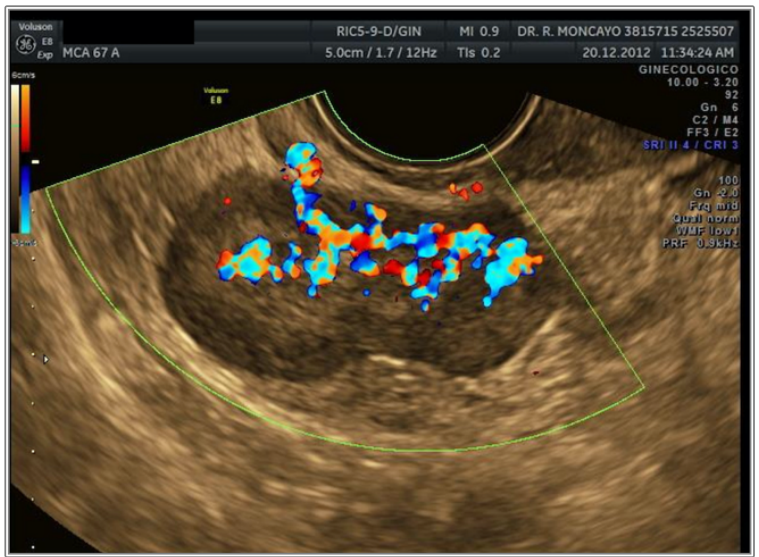

Figure 2. Doppler ultrasound showing abundant vascularization with a characteristic tree-shaped structure penetrating into the central part of the lesion.

Velocity measurement detected low impedance of intratumoral circulation, with a diminished vascular resistance $(\mathrm{RI}=0.4)$ and high mean velocity $(S / D=1.73)$ [Figure 3]. Additionally, an irregular suspicious mass, poorly defined with variable intratumoral echogenicity, was found on the anterior abdominal wall of the epigastrium suggesting a new locoregional recurrence of gastric carcinoma [Figure 4]. In March 2016, bilateral oophorectomy was performed and histopathology confirmed the diagnosis of Krukenberg tumors. One week later, the patient died due to acute mesenteric vein thrombosis. 


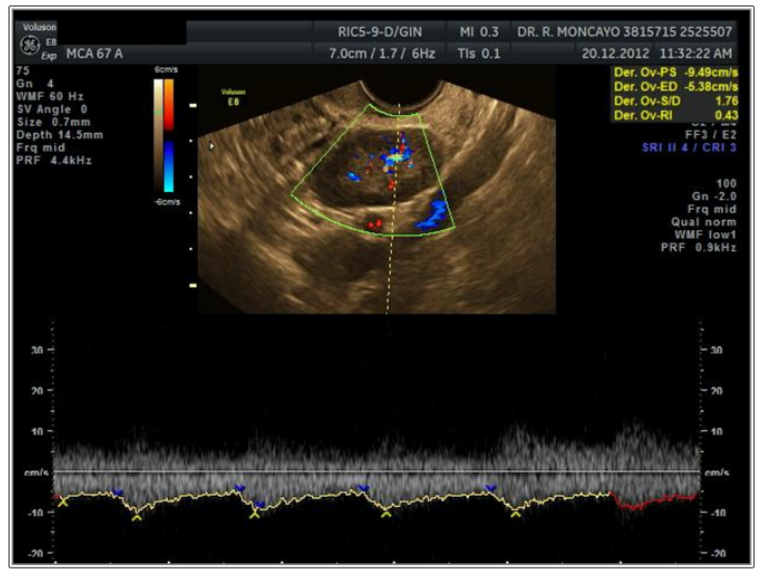

Figure 3. Doppler ultrasound showing the low impedance of intratumoral circulation in ovarian malignancy.

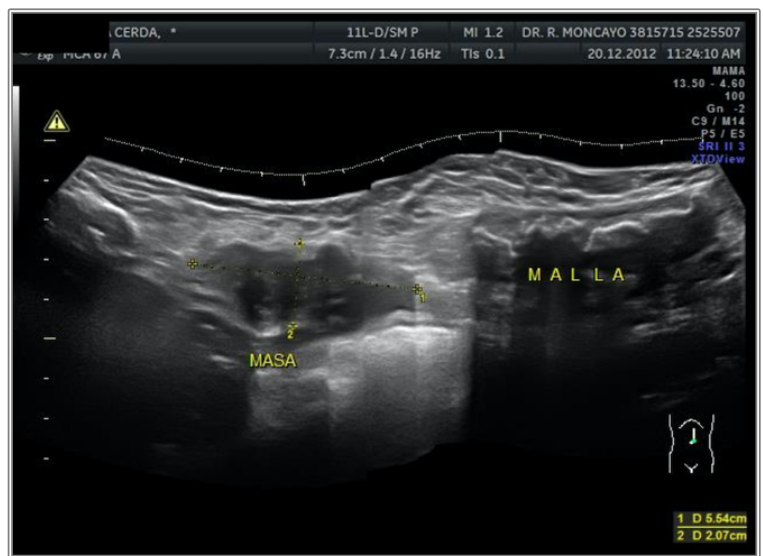

Figure 4. Ultrasound of the abdominal wall showing epigastric mass suspicious of locoregional recurrence of gastric carcinoma.

\section{Discussion}

Most of Kruckenberg metastases are diagnosed along with the identification of the primary tumor, but in $20-30 \%$ of cases, a conclusion can be made only after surgical removal of the neoplasm. According to many clinical and diagnostic features, metastatic ovarian tumors are similar to the primary tumors of the ovaries, which causes several difficulties in their detection and recognition and causes a high frequency of diagnostic confusions.

Kruckenberg's metastases are a consequence of cancer dissemination that originally occurred outside the ovaries. Up to $70 \%$ of all primary carcinomas are gastric and colorectal cancer.

Less commonly, Kruckenberg tumors arise due to the spread of carcinomas of the mammary gland (4\%), uterine body and cervix, appendix (3\%), biliary system (3\%), bladder, pancreas [6]. Sometimes the primary focus is not determined, it can be suspected just due to the presence of Krukenberg metastasis. In $80 \%$ of cases, secondary tumors are found in both ovaries and have sizes from 5 to $20 \mathrm{~cm}$ [7].

Ovarian metastasis has specific sonographic features and is characterized by the presence of asymmetrically enlarged bilateral encapsulated masses with a solid heterogeneous structure, smooth surface and ascites. Choi et al. [3] described varied echogenicity within 16 Krukenberg tumors on ultrasound examination: solid in six patients, mixed in six and predominantly cystic in others. Shimizu et al. [8] reported that most Krukenberg tumors had clear tumor margins, irregular hyperechoic solid patterns, and "moth-eaten" cyst formations on real-time grayscale sonography; while primary ovarian cancers had unclear tumor margins, an irregular hypoechoic solid pattern, clear cyst formation with papillary growths and uneven thickness of the septum.

The echostructure of Krukenberg tumors is usually homogeneously hyperechoic. On color Doppler examination, abundant vascularization is detected; in particular, as in the present case, the main vessel with a tree-shaped structure penetrating from the periphery into the central part of the lesion is usually observed [9].

Various blood velocity measurements have been used to show the low impedance of intratumoral circulation in ovarian malignancy. Chen et al. [10] reported that the resistance index was significantly lower in patients with metastatic ovarian carcinoma than in patients with 
primary ovarian carcinoma. Active angiogenesis might be the key factor in these findings. The prognosis for the patients with Krukenberg tumor is extremely poor, the survival rate is $12-23.4 \%$ and on average does not exceed 1.5 years [11].

Among various localizations of Kruckenberg's tumors, breast cancer prognostically proceeds more favorably. The worst prognosis and progressive course of the disease are characteristic of gastric carcinoma.

Peng et al. performed a retrospective analysis of treatment outcomes for 133 patients with Kruckenberg tumors arising from the stomach [12]. The authors concluded that ovarian metastasectomy can have a positive effect on survival in some patients. In the absence of ascites and in case gastric cancer resection performed, the authors recommend considering the patient as a potential candidate for ovarian metastasectomy. If the primary tumor of the stomach has not been removed or cannot be technically removed and ascites is diagnosed, surgical treatment is not practical [12,13].

In conclusion, usually, Krukenberg tumors are bilateral, and we report a solid tumor with variable echogenic density and abundant vascularization on color Doppler examination. Although there is no effective treatment for these tumors, except for complete resection of both primary and ovarian lesions, chemotherapy is of limited benefit. Hence prophylactic bilateral oophorectomy is recommended for women with stomach cancer.

\section{Conflict of interest}

All authors declare that they have no conflict of interest.

\section{Funding}

There was no funding received for this paper.

\section{References}

1. Krukenberg F. Ueber das Fibrosarcoma ovarii mucocellulare (Carcinomatodes). Archiv für Gynäekologie 1896;50:287-321.

2. Woodruff JD, Novac ER. Krukenberg tumor: a study of 48 cases from the ovarian tumor registry. Obstet Gynecol 1960;15:351-9.

3. Choi BI, Choo IW, Han MC, Kim CW. Sonographic appearance of Krukenberg tumor from gastric carcinoma. Gastrointest Radiol 1988;13:15-8.

4. Jiang R, Tang J, Cheng X, Zang RY. Surgical treatment for patients with different origins of Krukenberg tumors: Outcomes and prognostic factors. Eur J Surg Oncol 2009;35:92-7.

5. Kiyokawa T, Young RH, Scully RE. Krukenberg tumors of the ovary: a clinicopathologic analysis of 120 cases with emphasis on their variable pathologic manifestations. Am J Surg Pathol 2006;30:277-99.

6. Parkin DM, Bray F, Ferlay J, Pisani P. Global cancer statistics, 2002. CA Cancer J Clin 2005;55:74-108.

7. Hiremath R, Prasanti PK, Maesh, Pailoor A. Bilateral Krukenberg tumours diagnosed primarily by transabdominal sonography- a case report. J Clin Diagn Res 2015;9:TD01-TD03.

8. Shimizu H, Yamasaki M, Ohama K, Tadaharu N, Tanaka Y. Characteristic ultrasonographic appearance of the Krukenberg tumor. J Clin Ultrasound 1990;18:697-703.

9. Testa AC, Mancari R, Di Legge A. The "lead vessel”: a vascular ultrasound feature of metastasis in the ovaries. Ultrasound Obstet Gynecol 2008;31:21821.

10. Chen CY, Wu YC, Yen MS, Hung JH, Yuan CC, Chao KC. The power Doppler velocity index pulsatility index, and resistive index can assist in making a differential diagnosis of primary ovarian carcinoma and Krukenberg tumors. J Ultrasound Med 2007;26:921-26.

11. Testa CA, Licameli A, Legge AD, Mascilini F, Pelagalli M, Scambia G, et al. Colour Doppler sonographic features of Krukenberg tumour in pregnancy. J Ultrasound Med 2009;28:695-98.

12. Peng W, Hua RX, Jiang R. Surgical treatment for patients with Krukenberg tumor of stomach origin: clinical outcome and prognostic factors analysis. PLoS One 2013;8:e68227.

13. Das S, Sahu D, Wani M, Kummar RP. A curious discourse of Krukenberg tumor: a case report. J Gastrointest Oncol 2014;5:117-20. 